\title{
Interaction Between LncRNA and UPF1 in Tumors
}

\author{
Junjian $\mathrm{He}^{1,2}$ and Xiaoxin $\mathrm{Ma}^{1,2 *}$ \\ ${ }^{1}$ Department of Obstetrics and Gynecology, Shengjing Hospital of China Medical University, Shenyang, China, ${ }^{2}$ Key \\ Laboratory of Maternal-Fetal Medicine of Liaoning Province, Key Laboratory of Obstetrics and Gynecology of Higher \\ Education of Liaoning Province, Shenyang, China
}

Long non-coding RNAs (LncRNAs) can bind to other proteins or RNAs to regulate gene expression, and its role in tumors has been extensively studied. A common RNA binding protein, UPF1, is also a key factor in a variety of RNA decay pathways. RNA decay pathways serve to control levels of particular RNA molecules. The expression of UPF1 is often dysregulated in tumors, an observation which suggests that UPF1 contributes to development of a variety of tumors. Herein, we review evidence from studies of fourteen IncRNAs interact with UPF1. The interaction between IncRNA and UPFI provide fundamental basis for cell transformation and tumorigenic growth.

\section{OPEN ACCESS}

Edited by:

Kathleen Boris-Lawrie, University of Minnesota Twin Cities,

United States

Reviewed by:

Olivier Bensaude,

École Normale Supérieure, France

Shardul Kulkarni,

Pennsylvania State University,

United States

Sarah Fritz,

National Heart, Lung, and Blood Institute (NHLBI), United States

*Correspondence:

Xiaoxin Ma maxiaoxin666@aliyun.com

Specialty section

This article was submitted to

$R N A$,

a section of the journal

Frontiers in Genetics

Received: 01 November 2020 Accepted: 10 February 2021 Published: 01 March 2021

Citation:

He J and Ma X (2021) Interaction

Between LncRNA and UPF1

in Tumors. Front. Genet. 12:624905.

doi: 10.3389/fgene.2021.624905
Keywords: LncRNA - long non-coding RNA, UPF1, RBP, tumor, post-transcriptional regulation of gene expression

\section{INTRODUCTION}

About $93 \%$ of the human genome is transcribed into RNA. Protein-coding genes account for only about $2 \%$ of RNAs, with the vast majority of transcripts being non-coding RNAs (ncRNAs) (Qian et al., 2019). Historically, ncRNA was considered to be junk, or the by-products of transcription. However, recent studies have revealed that ncRNAs are crucial to many biological and pathological processes (Guttman et al., 2009; Ma et al., 2013; Bhan and Mandal, 2015; Yao et al., 2019). Non-coding RNAs are classified as either long non-coding RNAs or small non-coding RNAs (Manna and Sarkar, 2020), depending upon the number of nucleotides involved. LncRNAs are more than 200 nucleotides in length, and lack protein-coding capability (Chekanova, 2015; Yao et al., 2019). The mechanisms by which IncRNAs influence gene expression can be roughly divided into cis-acting and trans-acting (Figure 1). There are three possible mechanisms for cis action: (1) lncRNA recruits transcription factors to a locus to regulate the expression of a nearby gene (Figure 1A); (2) regulation of nearby genes during lncRNA transcription and shearing (Figure 1B); and (3) regulation of nearby genes by the lncRNA promoter or original DNA of the locus (Figure 1C; Ma et al., 2013; Kopp and Mendell, 2018; Yao et al., 2019). There are also three possible mechanisms of trans action: (1) the lncRNA regulates the chromatin state and gene transcription in distant regions (Figure 1D); (2) the lncRNA regulates the nuclear structure and organization (Figure 1E); and (3) the lncRNA binds to one or more proteins or other RNAs to regulate their activity (Figure 1F; Ma et al., 2013; Kopp and Mendell, 2018; Yao et al., 2019). LncRNAs contribute to a wide range of biological processes because of their diverse mechanisms of action (Chen and Shan, 2020).

Currently, about 2,000 RNA-binding proteins (RBPs) have been identified in humans (Corley et al., 2020; Qin et al., 2020). RBPs have been recognized as key regulators of transcription and post-transcriptional processing (Janakiraman et al., 2018; Xu et al., 2019). A large body of work has shown that many RBPs have impacts on tumorigenesis and cancer cell survival (Gao et al., 2020; Schultz et al., 2020; Weiße et al., 2020). Recent research into ncRNAs has revealed the existence of complex RBP-ncRNA interactions (Jonas et al., 2020). 
A

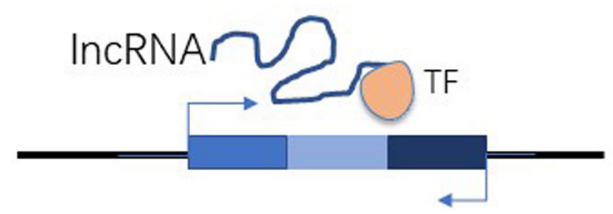

Gene X

B

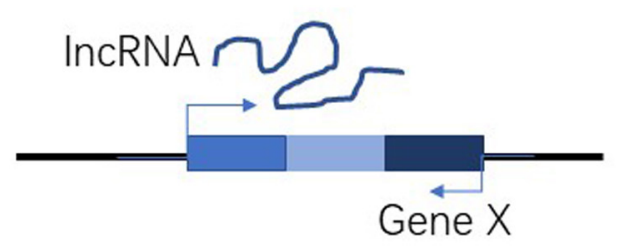

C

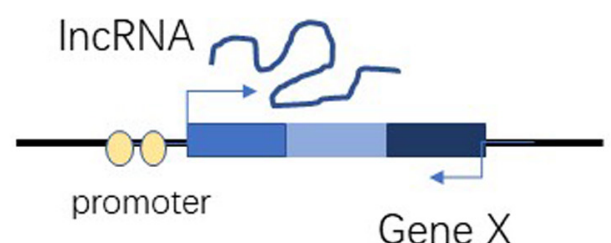

D

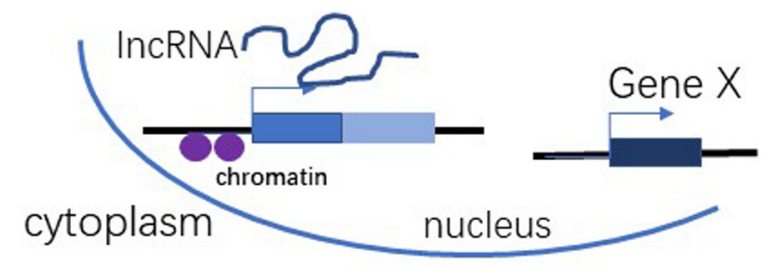

E

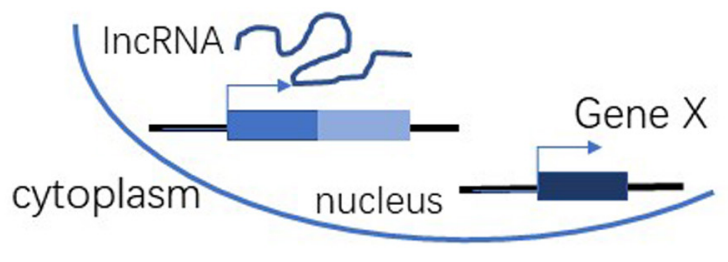

F

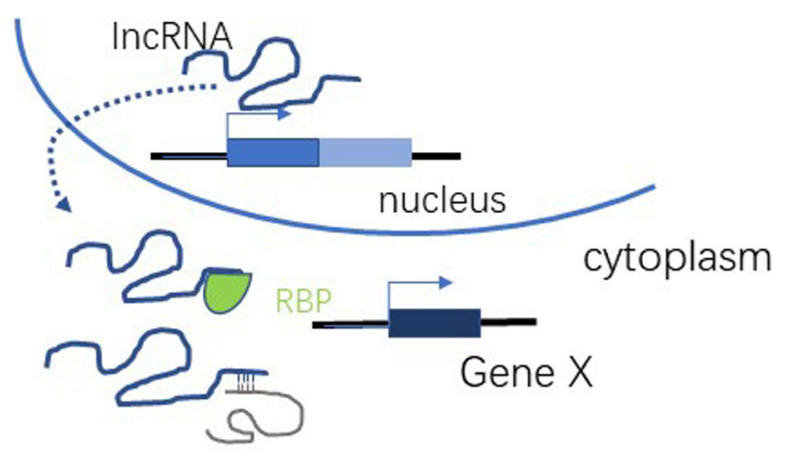

FIGURE 1 | Cis-acting and trans-acting roles of IncRNA. (A) LncRNA recruits transcription factors to a locus to regulate the expression of nearby genes. (B) Regulation of nearby genes during IncRNA transcription and shearing. (C) Regulation of nearby genes by IncRNA promoter or original DNA of the locus. (D) Regulation of chromatin state and gene transcription in distant regions. (E) Regulation of nuclear structure and organization. (F) Binding to proteins or other RNAs to regulate their activity.

Mechanistically, RBPs regulate RNA splicing, polyadenylation, mRNA stability, mRNA localization, and mRNA translation, by interacting with coding and non-coding RNAs and other proteins (Qin et al., 2020; Zang et al., 2020). LncRNAs can also affect the stability of RBPs (Zhang et al., 2020). The lncRNA PVT1 has no effect on the stability of MYC mRNA, but it can protect MYC protein from phosphorylation-mediated degradation in 8q24 amplified human cancer cells (Ferreri et al., 2010). The lncRNA GAS5 interacts with the WW domain of the YAP protein in colorectal cancer, and promotes the degradation of Yes1-related transcriptional regulators (YAP) through the ubiquitin-proteasome pathway (Ni et al., 2019).

UPF1 is a common RNA-binding protein, and is a critical molecule to the RNA decay pathway. The most common RNA decay pathways in which UPF1 participates are nonsensemediated mRNA decay (NMD) (Figure 2A) and staufen (STAU)-mediated mRNA decay (SMD) (Figure 2B) (Kim and Maquat, 2019). UPF1 plays a role in many other pathways, such as replication-dependent histone mRNA decay (HMD), glucocorticoid receptor-mediated mRNA decay
(GMD), regnase 1-mediated mRNA decay (RMD), and tudorstaphylococcal/micrococcal-like nuclease (TSN)-mediated microRNA decay (TumiD) (Kim and Maquat, 2019). In NMD, UPF1 binds to a premature termination codon (PTC) though translation termination complex, which consists of eRF1, eRF3, SMG1, SMG8, and SMG9 (Kurosaki and Maquat, 2016). PTCs are located more than 50 to 55 nucleotides upstream of an exon-exon junction, and exon junction complexes (EJCs) are 20 to 24 nucleotides upstream of an exon-exon junction (Hug et al., 2016; Kurosaki and Maquat, 2016). EJCs can be removed by ribosomes. However, the process will be stopped when there is a PTC (Kurosaki and Maquat, 2016). UPF2, together with UPF3 or UPF3X, binds to EJCs (Popp and Maquat, 2018). UPF1 then interacts with UPF2 and triggers the NMD pathway (Kurosaki and Maquat, 2016; Popp and Maquat, 2018; Kim and Maquat, 2019). UPF1 can mediate the degradation of NMD substrates through the phosphorylation/dephosphorylation cycle (Schoenberg and Maquat, 2012). NMD identifies and degrades mRNA which have PTCs, so that the synthesis of truncated proteins is prevented 

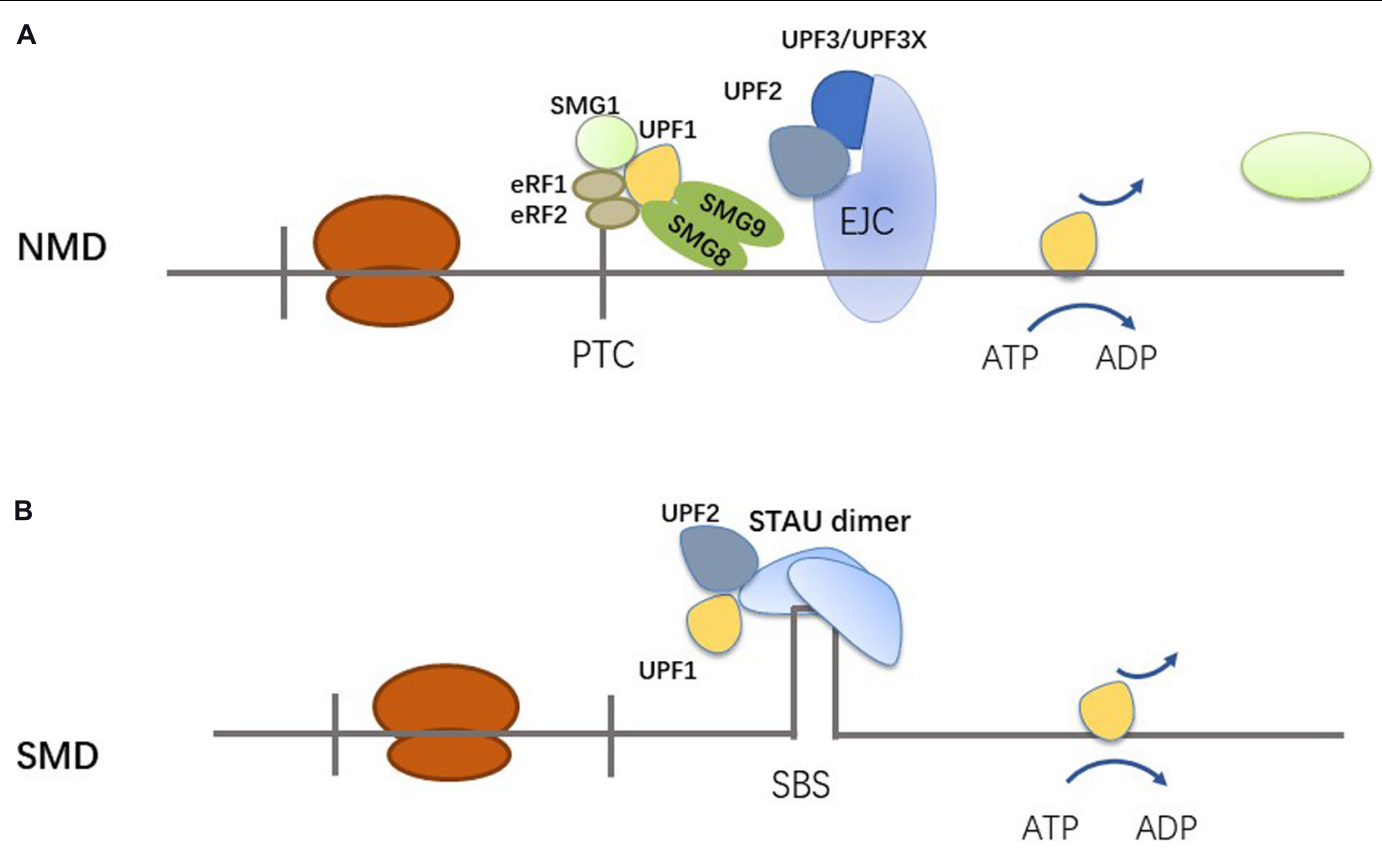

FIGURE 2 | Mechanisms of NMD and SMD. (A) UPF1 is engaged in NMD via binding PTC through the translation termination complex. (B) UPF1 is engaged in the SMD via 3' UTR-bound STAU1 or STAU2.

(Fatscher et al., 2015; Li et al., 2017; Kim and Maquat, 2019). Recent studies have shown that NMD not only degrades abnormal transcription products, but also regulates normal gene expression (Kurosaki et al., 2014; Kim and Maquat, 2019). The stability of $5-10 \%$ of normal physiological mRNAs are regulated by NMD (Kim and Maquat, 2019). The role of NMD in tumor development is complex. In some tumors, the expression of tumor suppressor genes is downregulated, because NMD selects specific mutations which cause destruction of tumor suppressor mRNAs, and tumor cells adjust their NMD activity to adapt to their microenvironment (Popp and Maquat, 2018). Mutations of the NMD machinery have been observed in tumors (Popp and Maquat, 2018). STAU1 binding sites (SBS) are a key trigger of SMD. There are two types of SBSs: one is a stem-loop structure formed by intramolecular base-paring within an mRNA $3^{\prime}$ UTR, and the other is formed by two Alu elements (Gong and Maquat, 2011; Park and Maquat, 2013). One of the two Alu elements comes from the mRNA of the SMD target, and the other Alu element comes from a lncRNA (Gong and Maquat, 2011). In SMD, STAU1 dimer binds to SBS and recruits and binds UPF2 together with UPF1, resulting in UPF1 phosphorylation and RNA degradation (Gong and Maquat, 2011; Plank and Wilkinson, 2018; Gowravaram et al., 2019). The SMD and NMD pathways share the steps of UPF1 phosphorylation. STAU1 and another NMD factor, UPF2, bind the overlapping region of the UPF1 $\mathrm{CH}$ domain, so that SMD and NMD are competing pathways (Park and Maquat, 2013).

In this review, we primarily discuss dysregulated lncRNAs in tumors, and the way in which these lncRNAs interact with UPF1 to regulate gene expression in tumors (Table 1).

\section{MECHANISMS OF LNCRNA INTERACTIONS WITH UPF1}

In order to understand the nature and effects of the interactions between lncRNA and UPF1, we can divide their mechanisms of action into the following four categories (Figure 3).

\section{LncRNAs Bind UPF1 and Affect the Expression of UPF1}

SNHG6 is upregulated in hepatocellular carcinoma (HCC) tissues and cell lines, in which it promotes the proliferation, invasion and migration of hepatocellular carcinoma cells, inhibits apoptosis, and induces cell cycle (Chang et al., 2016b). UPF1 is upregulated when SNHG6 is knocked down in human hepatocellular carcinoma cell lines (Chang et al., 2016b). SNHG6 is also upregulated in colorectal cancer (CRC) tissues and cell lines (Wang et al., 2019). The expression of UPF1 has an inverse correlation with SNHG6 in CRC tissues (Wang et al., 2019); when SNHG6 is knocked down, the expression of UPF1 is upregulated (Wang et al., 2019). These results indicate that SNHG6 influences the expression of UPF1. The lncRNA SNAI3-AS1 is upregulated in HCC tissues and cells (Li Y et al., 2019). Knockdown of SNAI3-AS1 can inhibit HCC cell invasion by upregulating the expression of UPF1 (Li Y et al., 2019). SNAI3-AS1 therefore reversely regulates the expression of UPF1 (Li Y et al., 2019).

\section{LncRNAs Bind UPF1 and Affect the Stability of mRNA}

The lncRNA ZFPM2-AS1 is upregulated in lung adenocarcinoma tissues and cell lines, promoting the 
TABLE 1 | LnCRNAs mentioned in the review.

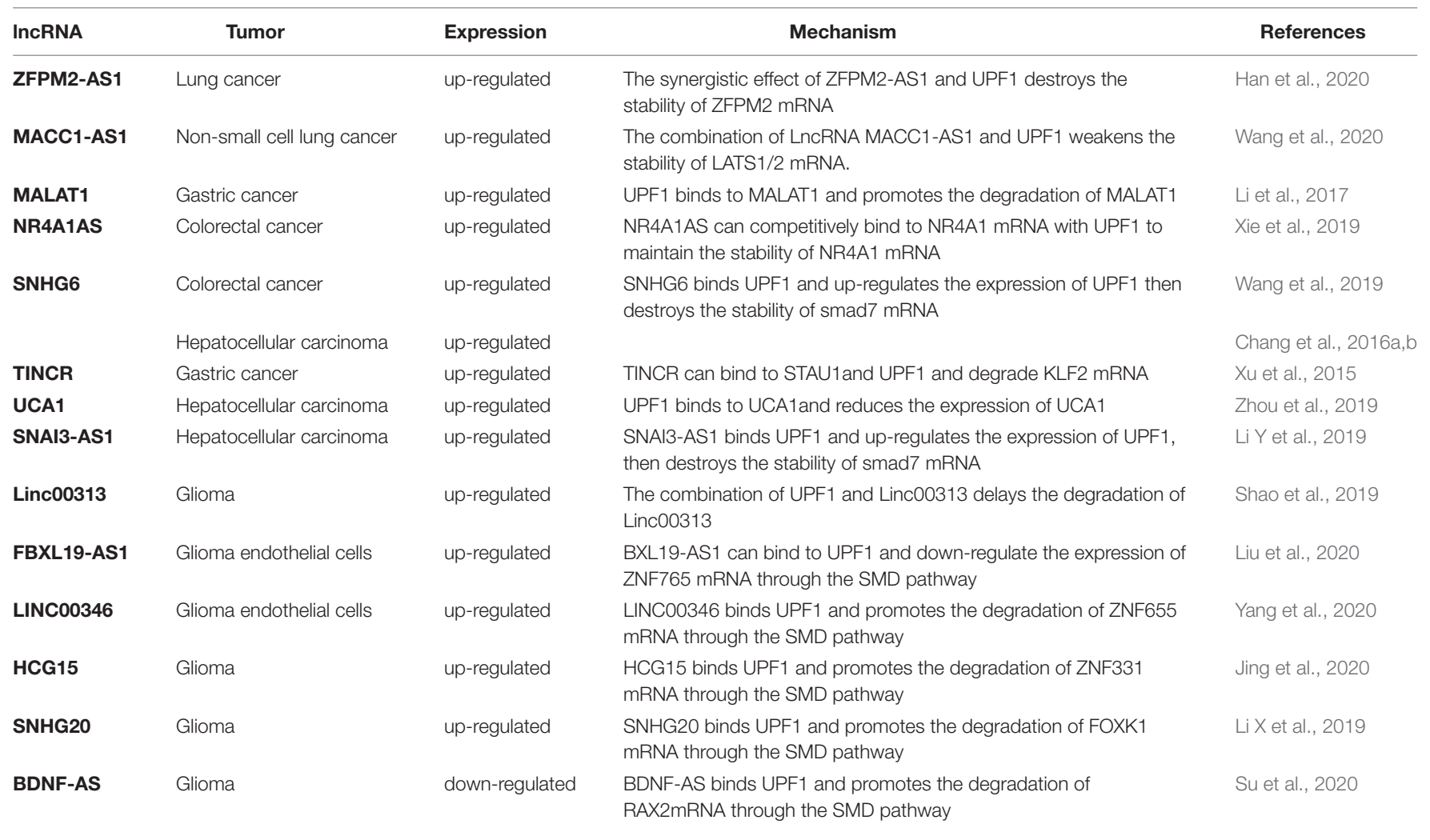

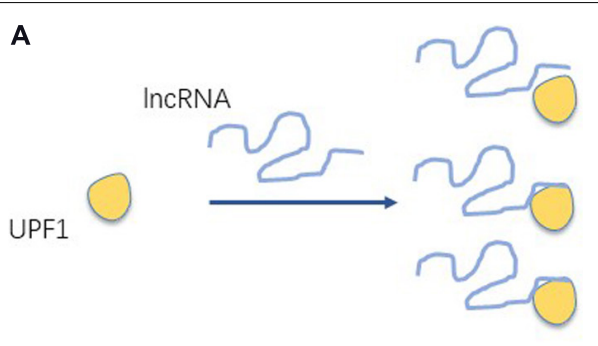

B

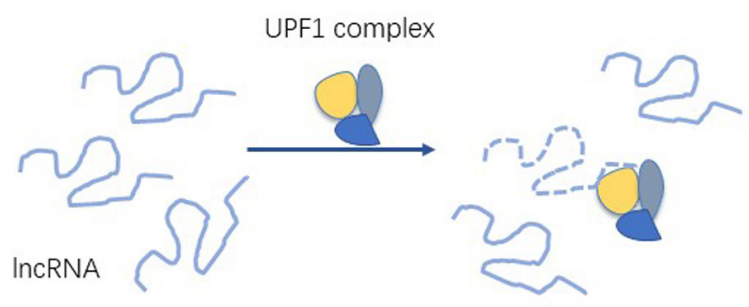

C

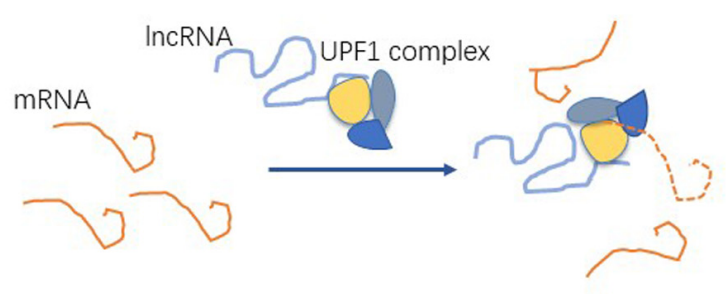

D

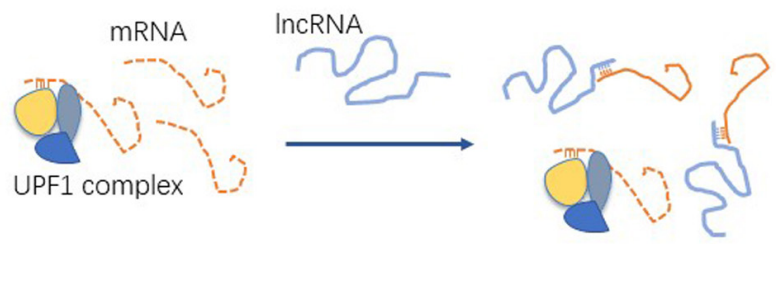

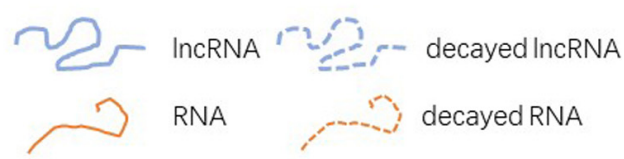

FIGURE 3 | Mechanisms of IncRNA interactions with UPF1. (A) IncRNAs bind UPF1 and affect the expression of UPF1. (B) IncRNAs bind UPF1 and affect the stability of mRNA. (C) UPF1 binds IncRNAs and affects the expression of IncRNAs. (D) UPF1 and IncRNA competitively bind mRNA. 
invasion and epithelial-mesenchymal transition (EMT) of lung adenocarcinoma cells (Han et al., 2020). ZFPM2, a gene located close to ZFPM2-AS1, is negatively regulated by LncRNA ZFPM2-AS1, and downregulated in lung adenocarcinoma tissues (Han et al., 2020). UPF1 participates in the regulation of ZFPM2-AS1 by ZFPM2. UPF1 can bind to the $3^{\prime}$ UTR region of ZFPM2-AS1 and ZFPM2 mRNA, and forms a binding complex in lung adenocarcinoma cells (Han et al., 2020). The synergistic effect of ZFPM2-AS1 and UPF1 destroys the stability of ZFPM2 mRNA, down-regulates the expression of ZFPM2, and promotes the proliferation, invasion, and EMT of lung adenocarcinoma cells (Han et al., 2020). The IncRNA MACC1-AS1 positively regulates the stemness of non-small cell lung cancer cells by downregulating the expression of LATS1/2, the key factor of the HIPPO pathway, to activate the HIPPO pathway (Wang et al., 2020). Both the lncRNA MACC1-AS1 and LATS1/2 mRNA can bind to UPF1 (Wang et al., 2020). Silencing UPF1 can attenuate the downregulation of LATS1/2 by overexpression of MACC1AS1 (Wang et al., 2020). The combination of MACC1-AS1 and UPF1 therefore weakens the stability of LATS1/2 mRNA (Wang et al., 2020). As previously mentioned, upregulation of SNHG6 reduces the expression of UPF1 in hepatocellular carcinoma and colorectal cancer (Chang et al., 2016b; Wang et al., 2019). Upregulated SNAI3-AS1 reversely regulates the expression of UPF1 in hepatocellular carcinoma (Li Y et al., 2019). Further studies have shown that SNHG6 and SNAI3-AS1 bind to UPF1 and promote the decay of smad7 mRNA (Chang et al., 2016a,b; Li Y et al., 2019). Smad7 is a negative regulator of the TGF- $\beta$ /SMAD pathway (Lukas et al., 2017). When Smad7 is downregulated, the TGF- $(\beta /$ SMAD pathway becomes active, and induces the EMT (Colak and Ten, 2017; Lukas et al., 2017). It has been reported that the expression of KLF2 protein is lower in gastric tumors, and KLF2 overexpression markedly enhanced cell apoptosis, and induced cell cycle arrest (Wang et al., 2017). The lncRNA TINCR is strongly upregulated in gastric cancer, and promotes the growth of gastric cancer cells by regulating the expression of KLF2 (Xu et al., 2015). RNA Immunoprecipitation (RIP) experiments confirmed that TINCR can bind to STAU1, and RNA pull-down assays revealed TINCR also binds to UPF1 (Xu et al., 2015). Further study confirmed that KLF2 mRNA, a target of SMD, was enriched by STAU1 antibody (Xu et al., 2015). STAU1 also binds to the $3^{\prime}$ UTR of KLF2 mRNA, and the KLF2 mRNA half-life was increased following the downregulation of STAU1 or TINCR (Xu et al., 2015). These results suggest that TINCR affects the expression of KLF2 through the SMD pathway (Xu et al., 2015). As a key factor of SMD, UPF1 interacts with a variety of lncRNAs, and plays an important role in tumorigenesis and the progression of glioma. The blood-tumor barrier (BTB) attenuates the efficacy of chemotherapy for glioma. The IncRNA FBXL19-AS1 is overexpressed in the cytoplasm of glioma microvessels and glioma endothelial cells (GECs) (Liu et al., 2020), and knockdown of FBXL19-AS1 increases the permeability of the BTB (Liu et al., 2020). Low expression of zinc finger protein 765 (ZNF765) in GEC improves the permeability of the BTB by inhibiting the promoter activity of tight junction-related proteins. Further studies have found that the binding of BXL19-AS1 and the $3^{\prime}$
UTR of ZNF765 mRNA produces an SBS (Liu et al., 2020). The half-life of ZNF765 mRNA was reduced when BXL19-AS1 was overexpressed, STAU1 was knocked down, or UPF1 was knocked down. BXL19-AS1 therefore affects the expression of ZNF765 mRNA through the SMD pathway (Liu et al., 2020). In GEC, LINC00346 is significantly increased, and the zinc finger protein 655 (ZNF655) is decreased (Yang et al., 2020). LINC00346 inhibition or ZNF655 overexpression hinder GEC angiogenesis (Yang et al., 2020). In terms of mechanism, LINC00346 and the 3' UTR of ZNF655 mRNA make up the SBS (Yang et al., 2020). RIP experiments showed that LINC00345 and ZNF655 mRNA both bind to STAU1 (Yang et al., 2020). When LINC00345, STAU1, or UPF1 was silenced, the half-life of ZNF655 mRNA was prolonged in GECs (Yang et al., 2020). LINC00345 therefore promotes the degradation of ZNF655 mRNA through SMD to promote angiogenesis in glioma (Yang et al., 2020). Similarly, the lncRNA HCG15 is highly expressed in gliomas, and promotes the degradation of ZNF331 mRNA through the SMD pathway. The lncRNA SNHG20 promotes the degradation of FOXK1 mRNA through the SMD pathway, thereby promoting the formation of glioma vascular mimicry and playing a role in promoting cancer (Li X et al., 2019; Jing et al., 2020). The down-regulation of the IncRNA BDNF-AS can promote the degradation of RAX2 mRNA via the SMD pathway, and inhibit the progression of malignancy in glioblastoma cells (Su et al., 2020).

\section{UPF1 Binds LncRNA and Affects the Expression of LncRNA}

MALAT1 is one of the most studied lncRNAs (Ji et al., 2003). MALAT1 is upregulated in a variety of tumors, including gastric cancer (Sun and Ma, 2019). MALAT1 promotes the proliferation, migration, invasion, and autophagy of gastric cancer by regulating the expression level of miRNAs (Malakar et al., 2017; YiRen et al., 2017; Li H et al., 2019; Lu et al., 2019). However, the expression of MALAT1 is regulated by UPF1. The expression of UPF1 is negatively correlated with the expression of MALAT1 in gastric cancer (Li et al., 2017). When UPF1 is overexpressed, the expression of MALAT1 is downregulated, and the half-life of MALAT1 is shortened (Li et al., 2017). UPF1 binds to MALAT1 and promotes its degradation, thereby inhibiting the expression of MALAT1 in gastric cancer (Li et al., 2017). In HCC, the expression of UPF1 is decreased, and silencing of UPF1 promotes the growth and invasion of HCC cells (Zhou et al., 2019). Knockdown of UPF1 in HCC cells increases the expression of UCA1 (Zhou et al., 2019). RIP experiments confirmed the combination of UPF1 (Zhou et al., 2019). The stability of UCA1 RNA was tested in HCC cells with UPF1 knocked down, and the decay rate of UCA1 was seen to increase (Zhou et al., 2019). Knockdown of UCA1 ameliorated the effect of UPF1 knockdown on HCC growth and invasion, indicating that UCA1 mediates the effect of UPF1 on the invasion and proliferation of hepatocellular carcinoma cells (Zhou et al., 2019). UPF1 and Linc00313 are both upregulated in glioma tissues and cells. Knocking down the expression of Linc00313 or UPF1 can inhibit the proliferation, invasion and migration of glioma cells, and promote 
apoptosis (Shao et al., 2019). RIP results revealed the combination of UPF1 and Linc00313 (Shao et al., 2019). When UPF1 is overexpressed, the half-life of Linc00313 is increased, and when UPF1 is knocked down, the opposite result was found (Shao et al., 2019). UPF1 appears to delay the degradation of Linc00313 and enhance the effect of Linc00313 on glioma (Shao et al., 2019).

\section{UPF1 and LncRNA Competitively Bind mRNA}

The upregulation of lncRNA NR4A1AS expression in colorectal cancer tissue is positively correlated with the expression of NR4A1 mRNA (Xie et al., 2019). NR4A1AS increases the stability of NR4A1 mRNA by forming RNA duplexes in CRC cells, thereby regulating the expression of NR4A1 (Xie et al., 2019). NR4A1 mRNA can also bind to UPF1, to increase its mRNA degradation rate (Xie et al., 2019). However, NR4A1AS cannot bind to UPF1. After silencing NR4A1AS in colorectal cancer cells, the NR4A1 mRNA bound to UPF1 was seen to almost double (Xie et al., 2019). These results indicate that NR4A1AS can bind to NR4A1 mRNA in competition with UPF1, to maintain the stability of NR4A1 mRNA in CRC cells and regulate the expression of NR4A1.

\section{DISCUSSION}

A considerable body of research shows that lncRNA is widely involved in tumorigenesis and the progression of various cancers. LncRNA regulates the expression and functions of other genes by binding to DNA, RNAs and proteins, and therefore participates in producing the tumor phenotype (Ramnarine et al., 2019; Cheng et al., 2020; Huang et al., 2020). As an RNA binding protein with multiple identities, UPF1 can participate in both the NMD pathway and the SMD pathway to regulate RNA stability and maintain homeostasis (Kim et al., 2007; Nasif et al., 2018). After UPF1 binds to lncRNA, the stability of the lncRNAs

\section{REFERENCES}

Bhan, A., and Mandal, S. S. (2015). LncRNA HOTAIR: a master regulator of chromatin dynamics and cancer. Biochim. Biophys. Acta 1856, 151-164. doi: 10.1016/j.bbcan.2015.07.001

Chang, L., Li, C., Guo, T., Wang, H., Ma, W., Yuan, Y., et al. (2016a). The human RNA surveillance factor UPF1 regulates tumorigenesis by targeting Smad7 in hepatocellular carcinoma. J. Exp. Clin. Cancer Res. 35:8.

Chang, L., Yuan, Y., Li, C., Guo, T., Qi, H., Xiao, Y., et al. (2016b). Upregulation of SNHG6 regulates ZEB1 expression by competitively binding miR-101-3p and interacting with UPF1 in hepatocellular carcinoma. Cancer Lett. 383, 183-194. doi: 10.1016/j.canlet.2016.09.034

Chekanova, J. A. (2015). Long non-coding RNAs and their functions in plants. Curr. Opin. Plant Biol. 27, 207-216.

Chen, H., and Shan, G. (2020). The physiological function of long-noncoding RNAs. Noncoding RNA Res. 5, 178-184. doi: 10.1016/j.ncrna.2020.09.003

Cheng, J., Meng, J., Zhu, L., and Peng, T. (2020). Exosomal noncoding RNAs in Glioma: biological functions and potential clinical applications. Mol. Cancer 19:66.

Colak, S., and Ten, D. P. (2017). Targeting TGF-beta signaling in cancer. Trends Cancer 3, 56-71. decreases in most cases. However, one study indicated that UPF1 binds to lncRNA and increases the stability of lncRNAs. These conflicting results seem to indicate a dual role for UPF1. The interactions between lncRNAs and UPF1 can regulate the degradation rate of other mRNAs. Antisense lncRNA may competitively bind to UPF1 to regulate mRNA expression. In some cases, the binding of lncRNA and UPF1 will affect the expression of UPF1, but the specific mechanism involved needs further exploration. Some studies have shown that after lncRNAs bind to RBPs, the stability of the RBPs is affected. LncRNA FAM83H-AS1 can bind to the HuR protein and promote its stability. However, the lncRNA MEG3 binds to the p-STAT3 protein, and promotes its degradation through ubiquitination (Dou et al., 2019; Zhang and Gao, 2019). Therefore, it appears that the binding of lncRNA and UPF1 may affect the stability of UPF1. Further exploration of the interactions and mechanisms underlying the relationship between lncRNA and UPF1 will help us to understand the influence of lncRNA and RBP on pathophysiological processes.

\section{AUTHOR CONTRIBUTIONS}

$\mathrm{JH}$ and XM contributed to the conception of the study, discussed, and improved the revised manuscript. JH wrote the manuscript. Both authors read and approved the final manuscript.

\section{FUNDING}

This work was supported by the National Natural Science Foundation of China (grant numbers 81872123 and 81472438), the Department of Science and Technology of Liaoning Province (grant number 2013225079), Shenyang City Science and Technology Bureau (grant number F14-158-9-47), and the Outstanding Scientific Fund of Shengjing Hospital (grant number 201601).

Corley, M., Burns, M. C., and Yeo, G. W. (2020). How RNA-binding proteins interact with RNA: molecules and mechanisms. Mol. Cell. 78, 9-29. doi: 10. 1016/j.molcel.2020.03.011

Dou, Q., Xu, Y., Zhu, Y., Hu, Y., Yan, Y., and Yan, H. (2019). LncRNA FAM83HAS1 contributes to the radioresistance, proliferation, and metastasis in ovarian cancer through stabilizing HuR protein. Eur. J. Pharmacol. 852, 134-141. doi: 10.1016/j.ejphar.2019.03.002

Fatscher, T., Boehm, V., and Gehring, N. H. (2015). Mechanism, factors, and physiological role of nonsense-mediated mRNA decay. Cell. Mol. Life Sci. 72, 4523-4544. doi: 10.1007/s00018-015-2017-9

Ferreri, A. J., Illerhaus, G., Zucca, E., Cavalli, F., and International Extranodal Lymphoma Study Group (2010). Flows and flaws in primary central nervous system lymphoma. Nat. Rev. Clin. Oncol. 7, $1-2$.

Gao, L., Meng, J., Zhang, Y., Gu, J., Han, Z., Wang, X., et al. (2020). Development and validation of a six-RNA binding proteins prognostic signature and candidate drugs for prostate cancer. Genomics 112, 4980-4992. doi: 10.1016/ j.ygeno.2020.08.034

Gong, C., and Maquat, L. E. (2011). IncRNAs transactivate STAU1-mediated mRNA decay by duplexing with 3'. UTRs via Alu elements. Nature 470, 284-288. doi: 10.1038/nature09701 
Gowravaram, M., Schwarz, J., Khilji, S. K., Urlaub, H., and Chakrabarti, S. (2019). Insights into the assembly and architecture of a Staufen-mediated mRNA decay (SMD)-competent mRNP. Nat. Commun. 10:5054.

Guttman, M., Amit, I., Garber, M., French, C., Lin, M. F., Feldser, D., et al. (2009). Chromatin signature reveals over a thousand highly conserved large noncoding RNAs in mammals. Nature 458, 223-227. doi: 10.1038/nature07672

Han, S., Cao, D., Sha, J., Zhu, X., and Chen, D. (2020). LncRNA ZFPM2AS1 promotes lung adenocarcinoma progression by interacting with UPF1 to destabilize ZFPM2. Mol. Oncol. 14, 1074-1088. doi: 10.1002/1878-0261. 12631

Huang, Z., Zhou, J. K., Peng, Y., He, W., and Huang, C. (2020). The role of long noncoding RNAs in hepatocellular carcinoma. Mol. Cancer 19:77.

Hug, N., Longman, D., and Caceres, J. F. (2016). Mechanism and regulation of the nonsense-mediated decay pathway. Nucleic Acids Res. 44, 1483-1495. doi: 10.1093/nar/gkw010

Janakiraman, H., House, R. P., Gangaraju, V. K., Diehl, J. A., Howe, P. H., and Palanisamy, V. (2018). The long (lncRNA) and short (miRNA) of it: TGFbetamediated control of RNA-binding proteins and noncoding RNAs. Mol. Cancer Res. 16, 567-579. doi: 10.1158/1541-7786.mcr-17-0547

Ji, P., Diederichs, S., Wang, W., Böing, S., Metzger, R., Schneider, P. M., et al. (2003). MALAT-1, a novel noncoding RNA, and thymosin beta4 predict metastasis and survival in early-stage non-small cell lung cancer. Oncogene 22, 8031-8041. doi: 10.1038/sj.onc. 1206928

Jing, F., Ruan, X., Liu, X., Yang, C., Wang, D., Zheng, J., et al. (2020). The PABPC5/HCG15/ZNF331 feedback loop regulates vasculogenic mimicry of glioma via STAU1-mediated mRNA decay. Mol. Ther. Oncolytics 17, 216-231. doi: 10.1016/j.omto.2020.03.017

Jonas, K., Calin, G. A., and Pichler, M. R. N. A. - (2020). Binding proteins as important regulators of long non-coding RNAs in cancer. Int. J. Mol. Sci. 21:2969. doi: 10.3390/ijms21082969

Kim, Y. K., Furic, L., Parisien, M., Major, F., DesGroseillers, L., and Maquat, L. E. (2007). Staufen 1 regulates diverse classes of mammalian transcripts. EMBO J. 26, 2670-2681. doi: 10.1038/sj.emboj.7601712

Kim, Y. K., and Maquat, L. E. (2019). UPFront and center in RNA decay: UPF1 in nonsense-mediated mRNA decay and beyond. RNA 25, 407-422. doi: 10.1261/ rna.070136.118

Kopp, F., and Mendell, J. T. (2018). Functional classification and experimental dissection of long noncoding RNAs. Cell 172, 393-407. doi: 10.1016/j.cell.2018. 01.011

Kurosaki, T., Li, W., Hoque, M., Popp, M. W., Ermolenko, D. N., Tian, B., et al. (2014). A post-translational regulatory switch on UPF1 controls targeted mRNA degradation. Genes Dev. 28, 1900-1916. doi: 10.1101/gad. 245506.114

Kurosaki, T., and Maquat, L. E. (2016). Nonsense-mediated mRNA decay in humans at a glance. J. Cell. Sci. 129, 461-467. doi: 10.1242/jcs.181008

Li, H., He, C., Wang, X., Wang, H., Nan, G., and Fang, L. (2019). MicroRNA-183 affects the development of gastric cancer by regulating autophagy via MALAT1miR-183-SIRT1 axis and PI3K/AKT/mTOR signals. Artif. Cells Nanomed. Biotechnol. 47, 3163-3171. doi: 10.1080/21691401.2019.1642903

Li, L., Geng, Y., Feng, R., Zhu, Q., Miao, B., Cao, J., et al. (2017). The human RNA surveillance factor UPF1 modulates gastric cancer progression by targeting long non-coding RNA MALAT1. Cell. Physiol. Biochem. 42, 2194-2206. doi: 10.1159/000479994

Li, X., Xue, Y., Liu, X., Zheng, J., Shen, S., Yang, C., et al. (2019). ZRANB2/SNHG20/FOXK1 axis regulates vasculogenic mimicry formation in glioma. J. Exp. Clin. Cancer Res. 38:68.

Li, Y., Guo, D., Ren, M., Zhao, Y., Wang, X., Chen, Y., et al. (2019). Long non-coding RNA SNAI3-AS1 promotes the proliferation and metastasis of hepatocellular carcinoma by regulating the UPF1/Smad7 signalling pathway. J. Cell. Mol. Med. 23, 6271-6282. doi: 10.1111/jcmm.14513

Liu, X., Wu, P., Su, R., Xue, Y., Yang, C., Wang, D., et al. (2020). IGF2BP2 stabilized FBXL19-AS1 regulates the blood-tumour barrier permeability by negatively regulating ZNF765 by STAU1-mediated mRNA decay. RNA Biol. 17, 1777-1788. doi: 10.1080/15476286.2020.1795583

Lu, Z., Luo, T., Pang, T., Du, Z., Yin, X., Cui, H., et al. (2019). MALAT1 promotes gastric adenocarcinoma through the MALAT1/miR-181a-5p/AKT3 axis. Open Biol. 9:190095. doi: 10.1098/rsob.190095
Lukas, D., Yogev, N., Kel, J. M., Regen, T., Mufazalov, I. A., Tang, Y., et al. (2017). TGF-beta inhibitor Smad7 regulates dendritic cell-induced autoimmunity. Proc. Natl. Acad. Sci. U.S.A. 114, E1480-E1489.

Ma, L., Bajic, V. B., and Zhang, Z. (2013). On the classification of long non-coding RNAs. RNA Biol. 10, 925-933.

Malakar, P., Shilo, A., Mogilevsky, A., Stein, I., Pikarsky, E., Nevo, Y., et al. (2017). Long noncoding RNA MALAT1 promotes hepatocellular carcinoma development by SRSF1 upregulation and mTOR activation. Cancer Res. 77, 1155-1167. doi: 10.1158/0008-5472.can-16- 1508

Manna, D., and Sarkar, D. (2020). Non-coding RNAs: regulating disease progression and therapy resistance in hepatocellular carcinoma. Cancers (Basel) 12:1243. doi: $10.3390 /$ cancers 12051243

Nasif, S., Contu, L., and Muhlemann, O. (2018). Beyond quality control: the role of nonsense-mediated mRNA decay (n.d.) in regulating gene expression. Semin. Cell Dev. Biol. 75, 78-87. doi: 10.1016/j.semcdb.2017.08.053

Ni, W., Yao, S., Zhou, Y., Liu, Y., Huang, P., Zhou, A., et al. (2019). Long noncoding RNA GAS5 inhibits progression of colorectal cancer by interacting with and triggering YAP phosphorylation and degradation and is negatively regulated by the $\mathrm{m}(6)$ A reader YTHDF3. Mol. Cancer 18:143.

Park, E., and Maquat, L. E. (2013). Staufen-mediated mRNA decay. Wiley Interdiscip. Rev. RNA 4, 423-435. doi: 10.1002/wrna.1168

Plank, T. D. M., and Wilkinson, M. F. (2018). RNA decay factor UPF1 promotes protein decay: a hidden talent. Bioessays 40:1700170 doi: 10.1002/bies. 201700170

Popp, M. W., and Maquat, L. E. (2018). Nonsense-mediated mRNA decay and cancer. Curr. Opin. Genet. Dev. 48, 44-50.

Qian, X., Zhao, J., Yeung, P. Y., Zhang, Q. C., and Kwok, C. K. (2019). Revealing Incrna structures and interactions by sequencing-based approaches. Trends Biochem. Sci. 44, 33-52. doi: 10.1016/j.tibs.2018.09.012

Qin, H., Ni, H., Liu, Y., Yuan, Y., Xi, T., Li, X., et al. (2020). RNA-binding proteins in tumor progression. J. Hematol. Oncol. 13:90.

Ramnarine, V. R., Kobelev, M., Gibb, E. A., Nouri, M., Lin, D., Wang, Y., et al. (2019). The evolution of long noncoding RNA acceptance in prostate cancer initiation, progression, and its clinical utility in disease management. Eur. Urol. 76, 546-559. doi: 10.1016/j.eururo.2019.07.040

Schoenberg, D. R., and Maquat, L. E. (2012). Regulation of cytoplasmic mRNA decay. Nat. Rev. Genet. 13, 246-259. doi: 10.1038/nrg3160

Schultz, C. W., Preet, R., Dhir, T., Dixon, D. A., and Brody, J. R. (2020). Understanding and targeting the disease-related RNA binding protein human antigen R (HuR). Wiley Interdiscip. Rev. RNA 11:e1581.

Shao, L., He, Q., Liu, Y., Liu, X., Zheng, J., Ma, J., et al. (2019). UPF1 regulates the malignant biological behaviors of glioblastoma cells via enhancing the stability of Linc-00313. Cell Death Dis. 10:629.

Su, R., Ma, J., Zheng, J., Liu, X., Liu, Y., Ruan, X., et al. (2020). PABPC1-induced stabilization of BDNF-AS inhibits malignant progression of glioblastoma cells through STAU1-mediated decay. Cell Death Dis. 11:81.

Sun, Y., and Ma, L. (2019). New insights into long non-coding RNA MALAT1 in cancer and metastasis. Cancers (Basel) 11:216. doi: 10.3390/cancers11020216

Wang, C., Li, L., Duan, Q., Wang, Q., and Chen, J. (2017). Kruppellike factor 2 suppresses human gastric tumorigenesis through inhibiting PTEN/AKT signaling. Oncotarget 8, 100358-100370. doi: 10.18632/oncotarget. 22229

Wang, X., Lai, Q., He, J., Li, Q., Ding, J., Lan, Z., et al. (2019). LncRNA SNHG6 promotes proliferation, invasion and migration in colorectal cancer cells by activating TGF-beta/Smad signaling pathway via targeting UPF1 and inducing EMT via regulation of ZEB1. Int. J. Med. Sci. 16, 51-59. doi: 10.7150/ijms. 27359

Wang, X., Yu, X., Wei, W., and Liu, Y. (2020). Long noncoding RNA MACC1-AS1 promotes the stemness of nonsmall cell lung cancer cells through promoting UPF1-mediated destabilization of LATS1/2. Environ. Toxicol. 35, 998-1006. doi: 10.1002/tox. 22936

Weiße, J., Rosemann, J., Krauspe, V., Kappler, M., Eckert, A. W., Haemmerle, M., et al. (2020). RNA-binding proteins as regulators of migration, invasion and metastasis in oral squamous cell carcinoma. Int. J. Mol. Sci. 21:6835. doi: $10.3390 /$ ijms 21186835

Xie, X., Lin, J., Liu, J., Huang, M., Zhong, Y., Liang, B., et al. (2019). A novel lncRNA NR4A1AS up-regulates orphan nuclear receptor NR4A1 expression by blocking 
UPF1-mediated mRNA destabilization in colorectal cancer. Clin. Sci. (Lond.) 133, 1457-1473. doi: 10.1042/cs20181061

Xu, T. P., Liu, X. X., Xia, R., Yin, L., Kong, R., Chen, W. M., et al. (2015). SP1-induced upregulation of the long noncoding RNA TINCR regulates cell proliferation and apoptosis by affecting KLF2 mRNA stability in gastric cancer. Oncogene 34, 5648-5661. doi: 10.1038/onc.2015.18

Xu, Y., Wu, W., Han, Q., Wang, Y., Li, C., Zhang, P., et al. (2019). New insights into the interplay between non-coding RNAs and RNA-binding protein HnRNPK in regulating cellular functions. Cells 8:62. doi: 10.3390/cells8010062

Yang, C., Zheng, J., Liu, X., Xue, Y., He, Q., Dong, Y., et al. (2020). Role of ANKHD1/LINC00346/ZNF655 feedback loop in regulating the glioma angiogenesis via staufen1-mediated mRNA decay. Mol. Ther. Nucleic Acids 20, 866-878. doi: 10.1016/j.omtn.2020.05.004

Yao, R. W., Wang, Y., and Chen, L. L. (2019). Cellular functions of long noncoding RNAs. Nat. Cell Biol. 21, 542-551.

YiRen, H., YingCong, Y., Sunwu, Y., Keqin, L., Xiaochun, T., Senrui, C., et al. (2017). Long noncoding RNA MALAT1 regulates autophagy associated chemoresistance via miR-23b-3p sequestration in gastric cancer. Mol. Cancer 16:174.

Zang, J., Lu, D., and Xu, A. (2020). The interaction of circRNAs and RNA binding proteins: an important part of circRNA maintenance and function. J. Neurosci. Res. 98, 87-97. doi: 10.1002/jnr.24356
Zhang, J., and Gao, Y. (2019). Long non-coding RNA MEG3 inhibits cervical cancer cell growth by promoting degradation of P-STAT3 protein via ubiquitination. Cancer Cell Int. 19:175.

Zhang, X. Z., Liu, H., and Chen, S. R. (2020). Mechanisms of long non-coding RNAs in cancers and their dynamic regulations. Cancers (Basel) 12:1245. doi: $10.3390 /$ cancers 12051245

Zhou, Y., Li, Y., Wang, N., Li, X., Zheng, J., and Ge, L. (2019). UPF1 inhibits the hepatocellular carcinoma progression by targeting long non-coding RNA UCA1. Sci. Rep. 9:6652. doi: 10.1038/s41598-01943148-z

Conflict of Interest: The authors declare that the research was conducted in the absence of any commercial or financial relationships that could be construed as a potential conflict of interest.

Copyright (C) $2021 \mathrm{He}$ and Ma. This is an open-access article distributed under the terms of the Creative Commons Attribution License (CC BY). The use, distribution or reproduction in other forums is permitted, provided the original author(s) and the copyright owner(s) are credited and that the original publication in this journal is cited, in accordance with accepted academic practice. No use, distribution or reproduction is permitted which does not comply with these terms. 